\title{
Morphological Filtering and Stochastic Modeling-Based Segmentation of Masses on Mammographic Images
}

\author{
H. $\mathrm{Li}^{1,2}$, K. J. R. $\mathrm{Liu}^{1}$, Y. Wang ${ }^{2}$, and S. C. B. $\mathrm{Lo}^{2}$ \\ ${ }^{1}$ Electrical Engineering Department and Institute for Systems Research \\ University of Maryland at College Park, College Park, Maryland 20742 \\ ${ }^{2}$ ISIS Center, Georgetown University Medical Center, Washington, D.C. 20007 *
}

\begin{abstract}
The objective of this study is to develop an efficient method to highlight the geometric characteristics of mass patterns, and isolate the suspicious regions which in turn provide the improved segmentation of suspected masses. In this work, a combined method of using morphological operations, finite generalized Gaussian mixture modeling, and contextual Bayesian relaxation labeling was developed to enhance and segment various mammographic contexts and textures. This method was applied to segment suspicious masses on mammographic images. The testing results showed that the proposed method can detect all suspected masses as well as high contrast objects and can be used as an effective pre-processing step of mass detection in computer-aided diagnosis systems.
\end{abstract}

\section{INTRODUCTION}

In recent years, some computer-aided diagnosis (CAD) schemes for mass detection and classification have been developed by several researchers [1], [2], [3], [4], [5], [6]. Since masses have different size and shape, some research works only focused on the detection of spiculated masses [5], [7], and the others were proposed to detect circumscribed masses [1]. In all automatic detection procedures, segmentation of suspected masses from breast tissue background is of great importance since all subtle masses should not be missed in the detecting process. The segmentation of suspected masses is difficult due to the variability of normal breast tissue and the lower contrast and ill-defined margins of masses. Therefore, some research works were directed towards to the classification of masses from local regions of interest selected manually by experienced radiologists [8], [9]. The results of all these CAD methods indicate that a high sensitivity rate can be achieved at the expense of higher false-positive detection. However, it is difficult to compare the relative performance of these methods because the reported performance strongly depends on the degree of subtlety of masses in the training and testing database. Also, the performance of algorithms highly depends on the accuracy and efficacy of the segmentation of suspected masses and the feature extraction based on the segmented regions.

A number of image processing techniques have been proposed to perform suspicious mass segmentation. Lai et al

*This work was supported in part by an U.S. Army Grant DAMD17-93-J-3007 and an NSF NYI Award MIP-9457397.
[1] have proposed using a modified median filter to reduce noise and to enhance mammograms followed by locating suspicious masses by template matching. Brzakovic et al [2] used thresholding and fuzzy pyramid linking for mass localization. Yin et al proposed using bilateral-subtraction to determine possible mass locations [3]. Bianchi et al used a segmentation method by calculating medians in the histogram of the image [10]. Some other investigators proposed using feature-based segmentation of suspected masses [5], [11]. Recently, Petrick et al [12] proposed a two stage adaptive density weighted contrast enhancement filtering technique along with edge detection and morphological feature classification for automatic segmentation of potential masses.

Stochastic model-based image segmentation is an efficient approach to segment objects which have low contrast and embed on inhomogeneous background. This approach is a technique for partitioning an image into distinctive meaningful regions based on the statistical properties of both gray-level and labeled images. Recently, this segmentation technique has received considerable attention for medical image pattern segmentation [13], [14]. However, a good segmentation result would depend on the suitable model selection for a specific image modality [15]. On the other hand, when the stochastic model is fixed, the segmentation result can also be improved by pattern-dependent enhancement techniques if the geometric characteristics of patterns is pre-defined. In this study, based on the analysis of the characteristic features of masses, we proposed using finite generalized Gaussian mixture (FGGM) model to model the histogram of mammograms which enhanced by morphological operations, then employed contextual Bayesian relaxation labeling (CBRL) technique to perform the segmentation of suspected masses.

\section{Methods}

The framework of our proposed segmentation approach is shown in Fig. 1. A detailed description follows.

\section{A. Mass Enhancement by Morphological Operation}

Based on the geometric properties of the contexts and textures in mammograms, we designed a morphological filtering-based enhancement algorithm which performs as follows [16]. The textures without the pattern information of interest are extracted by

$$
r(i, j)=\max (0,[f(i, j)-(f \circ B)(i, j)])
$$




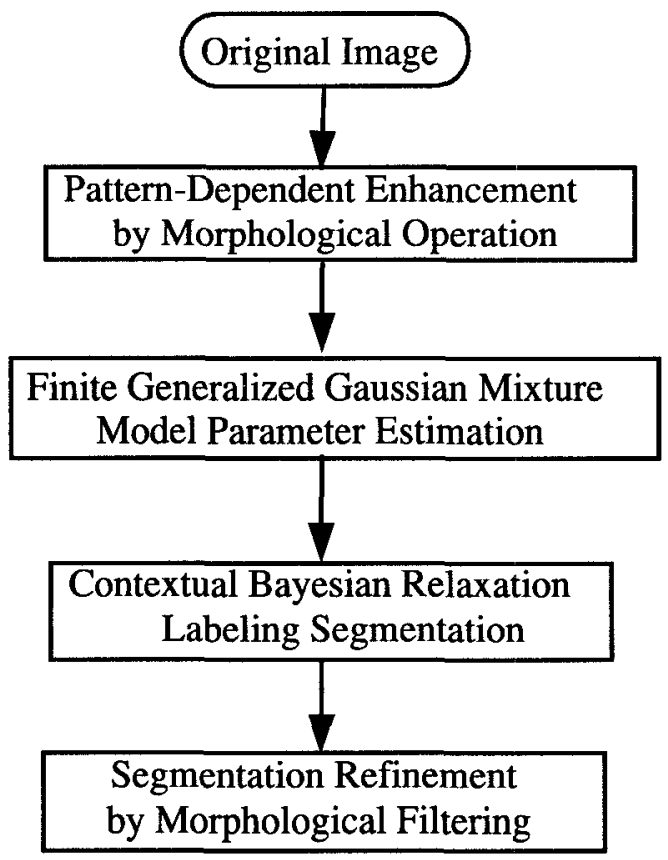

Fig. 1. The flow chart of the algorithm.

where $f(i, j)$ is the original image, $f \circ B$ is the morphological opening operation. $r(i, j)$ is the residue image between the original image and the opening of the original image by a specified structuring element $B$. The size of $B$ should be chosen smaller than the size of masses. Then, the regions of interests are enhanced by taking the difference between the original image and the specified rescaling transformation of the texture image

$$
f_{1}(i, j)=\max (0,[f(i, j)-g(r(i, j))])
$$

where $g(\cdot)$ is the specified rescaling transformation and $f_{1}(i, j)$ denotes the enhanced image. If we define $g(\cdot)$ as

$$
g(r(i, j))=r(i, j)+\left(f \circ B_{1}\right)(i, j)
$$

where $B_{1}$ is a specified structuring element which has large size than masses. This is equivalent to the following process. Let $r_{1}(i, j)$ be the mass pattern enhanced image by background correction, i.e.,

$$
r_{1}(i, j)=\max \left(0,\left[f(i, j)-\left(f \circ B_{1}\right)(i, j)\right]\right) .
$$

Then

$$
f_{1}(i, j)=\max \left(0,\left[r_{1}(i, j)-r(i, j)\right]\right) .
$$

We call it dual morphological operation. Fig. 2 shows the mass patch and the enhanced results after dual morphological operation. The one-dimensional profiles corresponding to each image patches in Fig. 2 is shown in Fig. 3. As we can see from Fig. 2, both background correction (Fig. 2 (c)) and dual morphological operation (Fig. 2 (d)) enhanced the mass pattern, but dual morphological operation removed more structural noise inside the mass region which in turn can improve the mass segmentation results. This phenomenon can also be clearly seen from Fig. 3 .

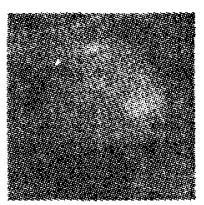

(a)

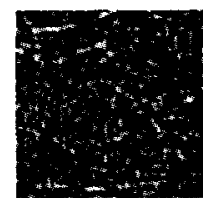

(b)

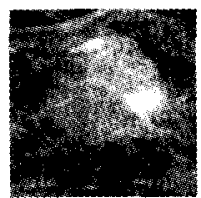

(c)

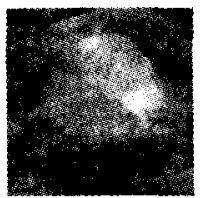

(d)
Fig. 2. The original and enhancement results of the mass patch using dual-morphological operation. (a) original image block $f(i, j)$; (b) the textures in image block without mass information $r(i, j)$; (c) the enhanced result by background correction $r_{1}(i, j)$; (d) the enhanced result by dual-morphological operation $f_{1}(i, j)$.

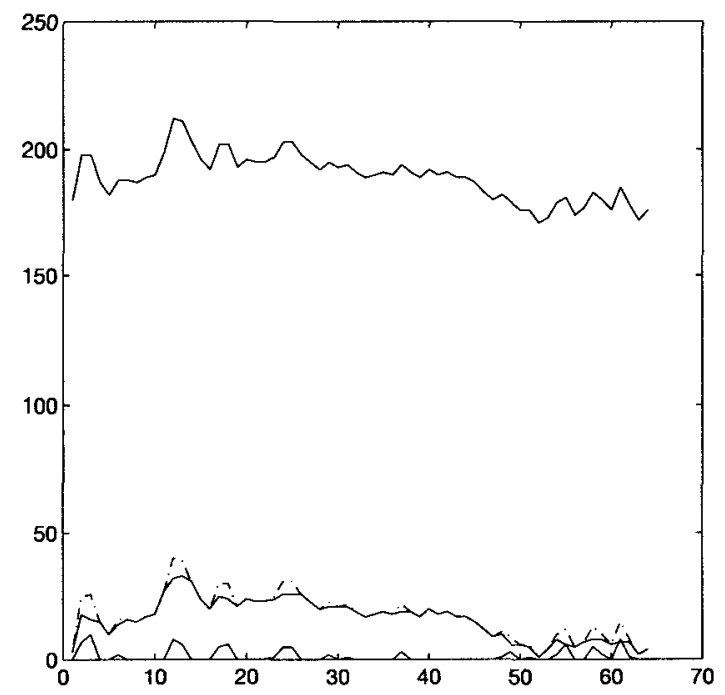

Fig. 3. The 1-D profiles of image patches in Fig. 2.

\section{B. Finite Generalized Gaussian Mixture Model}

Given an image which consists of $N_{1} \times N_{2}$ pixels, assume this image contains $K$ regions. By randomly reordering all pixels in the underlying probability space, one can treat pixel labels as random variables and introduce a new probability measure $\pi_{k}$. Then the finite generalize Gaussian mixture (FGGM) probability density function (pdf) of gray-level of each pixel is given by [15]:

$$
p\left(x_{i}\right)=\sum_{k=1}^{K} \pi_{k} p_{k}\left(x_{i}\right), \quad i=1, \cdots N_{1} N_{2}
$$

where $x_{i}$ is the gray-level of pixel $i, p_{k}\left(x_{i}\right)$ 's are conditional region pdf's with $\pi_{k}$, satisfying $\pi_{k}>0$, and $\sum_{k=1}^{K} \pi_{k}=1$. The generalized Gaussian pdf given region $k$ is defined by

$$
p_{k}\left(x_{i}\right)=\frac{\alpha \beta_{k}}{2 \Gamma(1 / \alpha)} \exp \left[-\left|\beta_{k}\left(x_{i}-\mu_{k}\right)\right|^{\alpha}\right], \quad \alpha>0
$$

where $\mu_{k}$ is the mean, $\Gamma(\cdot)$ is the Gamma function, and $\beta_{k}$ is a parameter related to the variance $\sigma_{k}$ by

$$
\beta_{k}=\frac{1}{\sigma_{k}}\left[\frac{\Gamma(3 / \alpha)}{\Gamma(1 / \alpha)}\right]^{1 / 2} .
$$

When $\alpha=2.0$, one has the Gaussian pdf; when $\alpha=1.0$, one has the Laplacian pdf. When $\alpha \gg 1$, the distribution tends to a uniform pdf; when $\alpha<1$, the pdf becomes 
sharp. Therefore, the generalized Gaussian model is suitable to model the histogram of those images which statistical properties are unknown.

The whole image can be well approximated by an independent and identically distributed random field $\mathbf{X}$. The corresponding joint pdf is

$$
P(\mathbf{x})=\prod_{i=1}^{N_{1} N_{2}} \sum_{k=1}^{K} \pi_{k} p_{k}\left(x_{i}\right)
$$

where $\mathbf{x}=\left[x_{1}, x_{2}, \cdots, x_{N_{1} N_{2}}\right]$, and $\mathbf{x} \in \mathbf{X}$.

The number of image regions $K$ in the FGGM model can be determined by Akaike information criterion (AIC), minimum description length (MDL), and minimum conditional bias and variance criterion (MCBV) approaches [17]. Once $K$ is known, one can initialize model parameters using adaptive Lloyd-Max histogram quantization algorithm [14] and estimate model parameters using expectation maximization (EM) algorithm [17].

\section{Contextual Bayesian Relaxation Labeling (CBRL)}

In this labeling task, one would need to define the localized FGGM model. Let $\partial i$ be the neighborhood of pixel $i$ with an $m \times m$ template centered at pixel $i$. An indicator function is used to represent the local neighborhood constraints $R_{i j}\left(l_{i}, l_{j}\right)=I\left(l_{i}, l_{j}\right)$, where $l_{i}$ and $l_{j}$ are labels of pixels $i$ and $j$, respectively. Note that pairs of labels are now either compatible or incompatible. Similar to reference [18], one can compute the frequency of neighbors of pixel $i$ which has the same label values $k$ as at pixel $i$

$$
\pi_{k}^{(i)}=p\left(l_{i}=k \mid \mathbf{l}_{\partial i}\right)=\frac{1}{m^{2}-1} \sum_{j \in \partial i, j \neq i} I\left(k, l_{j}\right)
$$

where $1_{\partial i}$ denotes the labels of the neighbors of pixel $i$. Since $\pi_{k}^{(i)}$ is a conditional probability of a region, the localized FGGM pdf of gray-level $x_{i}$ at pixel $i$ is given by

$$
p\left(x_{i} \mid \mathbf{l}_{\partial i}\right)=\sum_{k=1}^{K} \pi_{k}^{(i)} p_{k}\left(x_{i}\right)
$$

where $p_{k}\left(x_{i}\right)$ is given in (7). Assuming gray values of the image are conditional independent, the joint $\mathrm{pdf}$ of $\mathbf{x}$, given the context labels $\mathbf{1}$, is

$$
P(\mathbf{x} \mid \mathbf{l})=\prod_{i=1}^{N_{1} N_{2}} \sum_{k=1}^{K} \pi_{k}^{(i)} p_{k}\left(x_{i}\right)
$$

where $\mathbf{l}=\left(l_{i}: i=1, \cdots, N_{1} N_{2}\right)$.

It is known that CBRL algorithm can obtain a consistent labeling solution based on the localized FGGM model (11). Since $l$ represents the labeled image, it is consistent if $S_{i}\left(l_{i}\right) \geq S_{i}(k)$, for all $k=1, \cdots, K$ and for $i=1, \cdots, N_{1} N_{2}$ [18], where

$$
S_{i}(k)=\pi_{k}^{(i)} p_{k}\left(x_{i}\right)
$$

Now we can define

$$
A(\mathbf{l})=\sum_{i=1}^{N_{1} N_{2}}\left(\sum_{k} I\left(l_{i}, k\right) S_{i}(k)\right)
$$

as the average measure of local consistency, and

$$
L C_{i}=\sum_{k} I\left(l_{i}, k\right) S_{i}(k), \quad i=1, \cdots, N_{1} N_{2}
$$

represents the local consistency based on 1 . The goal is to find a consistent labeling 1 which can maximize (14). In the real application, each local consistency measure $L C_{i}$ can be maximized independently. In [18], it has been shown that when $R_{i j}\left(l_{i}, l_{j}\right)=R_{j i}\left(l_{j}, l_{i}\right)$, if $A(\mathrm{l})$ attains a local maximum at $\mathbf{l}$, then $\mathbf{l}$ is a consistent labeling.

Based on the localized FGGM model, $l_{i}^{(0)}$ can be initialized by ML classifier,

$$
l_{i}^{(0)}=\arg \left\{\max _{k} p_{k}\left(x_{i}\right)\right\}, \quad k=1, \cdots, K .
$$

Then, the order of pixels are randomly permutated and each label $l_{i}$ is updated to maximize $L C_{i}$, i.e., classify pixel $i$ into $k$ th region if

$$
l_{i}=\arg \left\{\max _{k} \pi_{k}^{(i)} p_{k}\left(x_{i}\right)\right\}, \quad k=1, \cdots, K
$$

where $p_{k}\left(x_{i}\right)$ is given in (7), $\pi_{k}^{(i)}$ is given in (10). For our specific mass segmentation, the brightest regions $(i=K)$ are selected as the suspicious masses.

\section{RESUlTS AND DISCUSSION}

Twenty real mammograms with masses were chosen as testing images. The areas of suspicious masses were located by an expert radiologist. The selected mammograms were digitized with an image resolution of $100 \mu m \times 100 \mu m$ per pixel by the laser film digitizer (Model: Lumiscan 150). The image sizes are $1792 \times 2560 \times 12 b p p$. For this study, we shrunk the digital mammograms with the resolution of $400 \mu \mathrm{m}$ by averaging $4 \times 4$ pixels into one pixel. This shrinking step is applicable for mass cases and can save computation time.

In order to justify the suitability of morphological structural elements, the geometric properties of the contexts and textures in mammograms were studied. At the resolution of $400 \mu m$, a disk with a diameter of 7 pixels was chosen as morphological structuring element $B$ to extract textures in mammograms. A disk with a diameter of 75 pixels was empirically chosen as morphological structuring element $B_{1}$ for background correction. In the last stage of our approach, we applied morphological opening and closing filtering using a disk with a diameter of 5 to eliminate small objects which contribute to false masses.

According to previous investigator's work [10], the suitable number of regions, $K$, is 8 for most mammograms. In this work, we used three information criteria (AIC, MDL, 
and MBVC) to determine $K$. Fig. 4 is one of the results based on one real mammogram. As we can see in Fig. 4, all three criteria achieved minimum when $K=8$ which is consistent with the previous research.

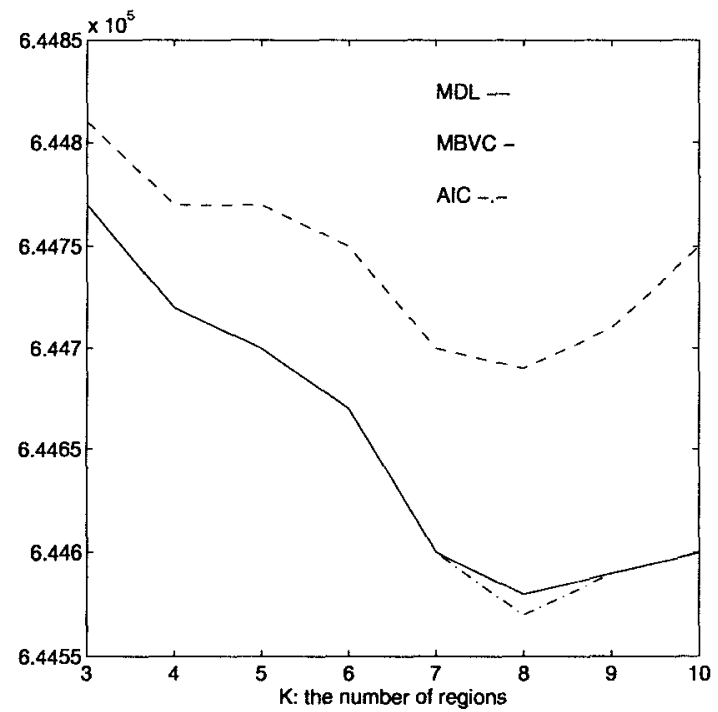

Fig. 4. The AIC, MDL, and MBVC curves with different number of region $K$

We fixed $K=8$, and changed the values of $\alpha$ for estimating the FGGM model parameters. We used global relative entropy (GRE) between the histogram and the estimated FGGM distribution as a measure of the estimation bias. We found that GRE achieved a minimum distance when the FGGM parameter $\alpha=3.0$ as shown in Fig. 5 . This indicated that the FGGM model is better than the finite normal mixture model $(\alpha=2.0)$, which has been mostly chosen in stochastic model-based segmentation, if the statistical properties of mammograms are not known.

With $K=8$, and $\alpha=3.0$, we compared the segmentation results based on the enhanced mammograms with those based on the original mammograms. The results demonstrated that all the areas of suspicious masses in our tested mammograms were detected after enhancement. On the other hand, only parts of suspicious masses were detected with the original mammograms. In addition, some very subtle cases were undetected based on original mammograms. The undetect areas were mainly occurred at lower intensity side of the shaded objects which, however, extracted on morphological enhanced mammograms. Fig. 6 is one of segmentation results with original and enhanced mammograms.

\section{CONCLUSIONS}

This work was a part of our research in mammographic mass detection. The experimental results indicate that the segmentation of suspected masses can be affected by different $K$ and $\alpha$. With suitable $K$ and $\alpha$, the segmentation results can be significantly improved by the proposed pattern-dependent enhancement algorithm using morpho-
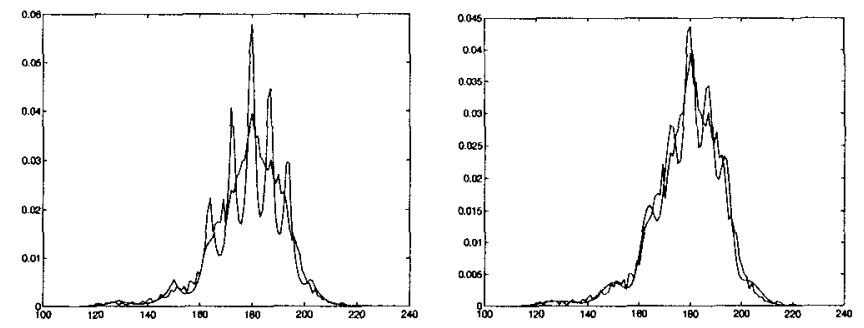

(a) $\alpha=1.0, G R E=0.0783$

(b) $\alpha=2.0, G R E=0.0369$
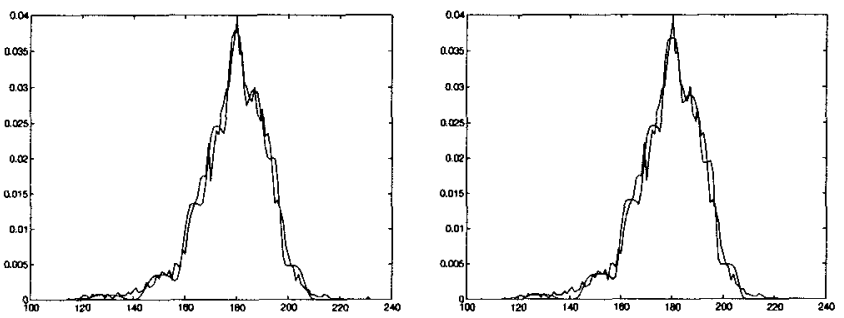

(c) $\alpha=3.0, G R E=0.0251$

(d) $\alpha=4.0, G R E=0.0282$

Fig. 5. The comparison of different learning curves and histogram of original mammogram, $K=8$.

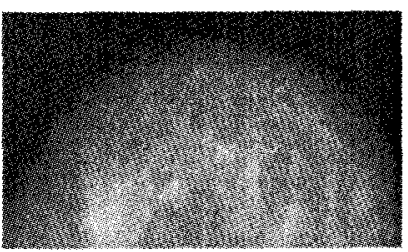

(a) Original Mammogram

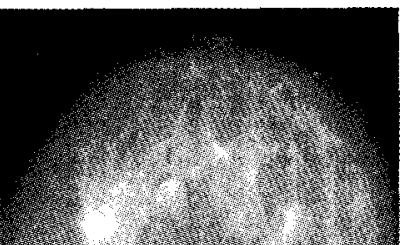

(c) Enhanced Mammogram

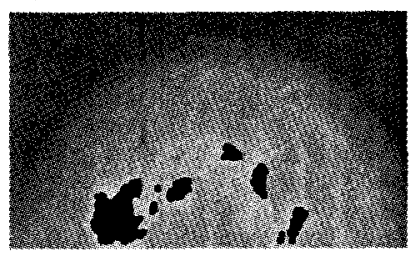

(b) Mass Segmentation

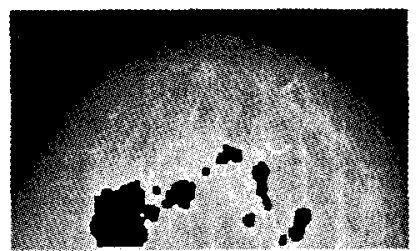

(d) Mass Segmentation
Fig. 6. The comparison of segmentation results between original and morphological enhanced mammograms, $K=8, \alpha=3.0$.

logical operations. Hence, morphological filtering combined with stochastic model-based segmentation is an effective way to extract mammographic suspicious patterns of interest, and thereby will facilitate the procedures of mammographic computer-aided diagnosis.

\section{REFERENCES}

[1] S. M. Lai, X. Li, and W. F. Bischof, "On Techniques for Detecting Circumscribed Masses in Mammograms," IEEE Trans. on Med. Imaging, Vol. 8, No. 4, pp. 377-386, 1989.

[2] D. Brzakovic, X. M. Luo, and P. Brzakovic, "An Approach to Automated Detection of Tumors in Mammograms," IEEE Trans. on Med. Imaging, Vol. 9, No. 3, pp. 233-241, 1990.

[3] F. F. Yin, M. L. Giger, C. J. Vyborny, K. Doi, and R. A. Schmidt, "Comparison of Bilateral-Subtraction and SingleImage Processing Techniques in the Computerized Detection of Mammographic Masses," Investigative Radiology, Vol. 28, No. 6, pp. 473-481, 1993.

[4] B. Zheng, Y. H. Chang, and D. Gur, "Computerized Detection of Masses in Digitized Mammograms Using Single-Image 
Segmentation and a Multilayer Topographic Feature Analysis," Acad. Radiology, Vol. 2, pp. 959-966, 1995.

[5] W. P. Kegelmeyer Jr., J. M. Pruneda, P. D. Bourland, A. Hillis, M. W. Riggs, and M. L. Nipper, "Computer-aided Mammographic Screening for Spiculated Lesions," Radiology, Vol. 191, pp. 331-337, 1994.

[6] H. D. Li, M. Kallergi, L. P. Clarke, V. K. Jain, and R. A. Clark, "Markov random Field for Tumor Detection in Digital Mammography," IEEE Trans. on Med. Imaging, Vol. 14, No. 43 pp. 565-576, 1995.

[7] M. Zhang and M. L. Giger, "Automated Detection of Spiculated Lesions and Architectural Distortions in Digitized Mammograms," Proc. SPIE, Vol. 2434, pp. 846-854, 1995.

[8] J. Kilday, F. Palmieri, and M. D. Fox, "Classifying Mammographic Lesions Using Computerized Image Analysis," IEEE Trans. on Med. Imaging, Vol. 12, No. 4, pp. 664-669, 1993.

[9] H. P. Chan, D. Wei, M. A. Helvie, B. Sahiner, D. D. Alder, M. M. Goodsitt, and N. Petrick, "Computer-Aided Classification of Mammographic Masses and Normal Tissue: Linear Discriminant Analysis in Texture Feature Space," Phys. Med. Biol., Vol. 40, pp. 857-876, 1995.

[10] M. J. Bianchi, A. Rios, and M. Kabuka, "An Algorithm for Detection of Masses, Skin Contours, and Enhancement of Microcalcifications in Mammograms," Proc. , Symposium for Computer Assisted Radiology, pp. 57-64, Winston-Salem, June 1994.

[11] D. H. Davies and D. R. Dance, "Computer Detection of Soft Tissue Masses in Digital Mammograms: Automatic Region Segmentation," Proc. SPIE, Vol. 1652, pp. 514-521, 1992.

[12] N. Petrick, H. P. Chan, B. Sahiner, and D. Wei, "An Adaptive Density-Weighted Contrast Enhancement Filter for Mammographic Breast Mass Detection," IEEE Trans. on Med. Imaging, Vol. 15, No. 1, pp. 59-67, 1996.

[13] T. Lei and W. Sewchand, "Statistical Approach to X-Ray CT Imaging and Its Application in Image Analysis-Part II: A New Stochastic Model-Based Image Segmentation Technique for XRay CT Image," IEEE Trans. on Med. Imaging, Vol. 11, No. 1, pp. 62-69, March 1992.

[14] Y. Wang and T. Adall, "Efficient Learning of Finite Normal Mixtures for Image Quantification," Proc. IEEE ICASSP, pp. 3422-3425, Atlanta, May 7-11, 1996.

[15] J. Zhang and J. W. Modestino, "A Model-Fitting Approach to Cluster Validation with Application to Stochastic Model-Based Image Segmentation," IEEE Trans. on PAMI, Vol. 12, No. 10, pp. 1009-1017, October 1990.

[16] H. Li, K. J. R. Liu, and S. C. Lo, "Nonlinear Filtering Enhancement and Histogram Modeling Segmentation of Masses for Digital Mammograms," Proc. 18th Annual International Conference of IEEE Engineering in Medicine and Biology Society, Amsterdam, The Netherlands, 1996.

[17] Y. Wang, "Image Quantification and the Minimum Condition Bias/Variance Criterion," Proc. 3oth Conf. Info. Sci. Sys., pp. 1061-1064, Princeton, March 20-22, 1996.

[18] R. A. Hummel and S. W. Zucker, "On the Foundations of Relaxation Labeling Processes," IEEE Trans. on PAMI, Vol. 5, No. 3, pp. 267-286, May 1983. 